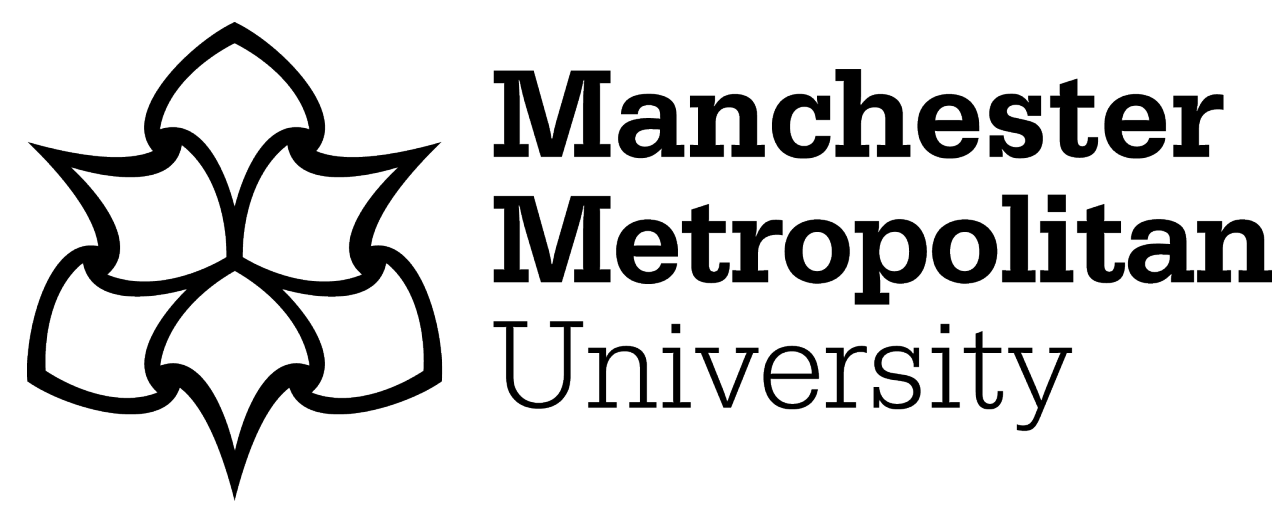

Wilkinson, Ben, Trick, Leanne, Knight, Annie, Valton, Vincent, Goodhand, James, Kennedy, Nick A, Heerasing, Neel, Ahmad, Tariq, Bland, Amy, Elliott, Rebecca, Roiser, Jonathan P and Dickens, Chris (2019) Factors associated with depression in people with inflammatory bowel disease: The relationship between active disease and biases in neurocognitive processing. Neurogastroenterology \& Motility, 31 (8). ISSN 1350-1925

Downloaded from: https: //e-space.mmu.ac.uk/625555/

Version: Accepted Version

Publisher: Wiley

DOI: https://doi.org/10.1111/nmo.13647

Please cite the published version 


\title{
Factors associated with depression in people with inflammatory bowel disease: The relationship between active disease and biases in neurocognitive processing
}

\author{
Ben Wilkinson $^{1}$ | Leanne Trick $^{1}$ | Annie Knight ${ }^{2}$ | Vincent Valton ${ }^{3}$ | James Goodhand ${ }^{4}$ \\ Nick A. Kennedy ${ }^{4}$ | Neel Heerasing ${ }^{4}$ | Tariq Ahmad ${ }^{4}$ | Amy Bland ${ }^{5}$ | Rebecca Elliott ${ }^{5}$ | \\ Jonathan P. Roiser $^{3}$ | Chris Dickens ${ }^{1}$
}

${ }^{1}$ Mental Health Research Group, College of Medicine and Health, University of Exeter, Exeter, UK

${ }^{2}$ Institute of Biomedical and Clinical Sciences, College of Medicine and Health, University of Exeter, Exeter, UK

${ }^{3}$ Institute of Cognitive

Neuroscience, University College London, London, UK

${ }^{4}$ Exeter IBD Research Group, Royal Devon and Exeter NHS Foundation Trust, Exeter, UK

${ }^{5}$ Division of Neuroscience \& Experimental Psychology, University of Manchester,

Manchester, UK

\section{Correspondence}

Chris Dickens, Room 1.04, College House, St Luke's Campus, Heavitree Road, Exeter, EX1 2LU, UK.

Email: c.m.dickens@exeter.ac.uk

\section{Funding information}

The submitted research was funded by the College of Medicine and Health, University of Exeter.

\begin{abstract}
Background: Depression is common among people with inflammatory bowel disease (IBD), though the causes remain unclear. We conducted a cross-sectional study to investigate the role of emotional processing biases in contributing to depression among people with IBD.

Materials and Methods: One hundred and twenty outpatients with IBD were recruited and: (a) completed questionnaires to record: age, sex, social support, socioeconomic status, anxiety and depression $(n=104)$, (b) underwent assessments of biases in emotional recognition ( $n=112)$, emotional memory and reinforcement learning (c) had recorded from clinical records: type of IBD, duration of IBD, IBD activity and (d) provided blood for high-sensitivity C-reactive protein levels ( $n=99)$.

Key Results: Sixty-eight participants had Crohn's disease and 49 had ulcerative colitis. Of these, 35 had active disease and 26 had depression. Those with depression were more likely to be female, lack social support, have active disease, be taking corticosteroids but not TNF-alpha inhibitors and exhibit less positive emotional recognition bias. On multivariable regression analysis, depression was associated independently with lack of social support (unstandardized regression coefficient $(B)=-1.40, P=0.02)$ and increased disease activity $(B=1.29, P=0.03)$. Causal steps analysis was consistent with less positive emotional recognition bias partially mediating the effects of disease activity on depression.

Conclusions and inferences: This is the first study to demonstrate links between disease activity and less positive biases in emotional recognition that could explain higher rates of depression among people with active IBD. Future prospective studies are required to confirm the effects of emotional processing biases in depression and allow stronger causal inferences to be drawn.
\end{abstract}

KEYWORDS

cognitive functioning, depression, inflammation, inflammatory bowel disease 


\section{1 | INTRODUCTION}

Depression affects $14 \%-27 \%$ patients with inflammatory bowel disease (IBD), which is approximately 2-3 times the prevalence in people without IBD. ${ }^{1-3}$ Depression in IBD is important because it is associated with more gastrointestinal symptoms independent of disease severity, ${ }^{4}$ worse health-related quality of life,${ }^{5-8}$ increased healthcare utilisation, ${ }^{9-11}$ and possibly relapses in disease activity. ${ }^{12-16}$ Depression is associated with a number of sociodemographic, clinical and psychological factors, ${ }^{1,13,17-21}$ though many of these risk factors are inter-related, and the main causes of depression among people with IBD remain unclear.

Recently, there has been considerable interest in the role of inflammation in depression. Observational studies in healthy and clinical populations have shown that inflammation is associated with depression. ${ }^{22-24}$ Also, controlled, experimental studies in healthy individuals have shown that acute inflammation causes short term increases in depressive symptoms. ${ }^{25,26}$ Among people with severe Crohn's disease, treatment with the anti-TNF-alpha drugs infliximab and adalimumab has been associated with a rapid reduction in depression, not attributable solely to reductions in clinical disease activity. ${ }^{27-29}$ However, it is unclear how inflammation causes depression. We postulate here that the effects of inflammation may be mediated via negative cognitive biases, particularly biases in the processing of emotionally salient information (henceforth emotional processing). ${ }^{30}$ Such negative cognitive biases are considered central to the development of depression, though their association with chronic inflammation in people with IBD has not been investigated previously. ${ }^{31,32}$

We conducted a cross-sectional study among hospital outpatients with IBD to identify sociodemographic, IBD-related and psychological factors that were independently associated with depression, and to explore whether negative biases in emotional processing mediated links between IBD activity and depression.

We tested the following hypotheses among outpatients with IBD:

1. Depression would be independently predicted by socio-demographic characteristics (age, sex, socioeconomic status, social support), medical characteristics (type of IBD, IBD activity), and psychological characteristics (negative biases in emotional processing)

2. Negative biases in emotional processing would mediate the effects of disease activity on depression

\section{2 | MATERIALS AND METHODS}

\section{1 | Subjects}

We recruited adults with known IBD attending the gastroenterology outpatients and biologic infusion clinics at the Royal Devon and

\section{Key Points}

- Depression is common in people with inflammatory bowel disease (IBD), but the actual causes of depression in this group are unknown.

- We found that depression was independently associated with increased IBD activity, and that less positive cognitive bias part-mediated the effects of disease activity on depression.

- This is the first study to demonstrate links between disease activity and less positive biases in emotional recognition that could explain higher rates of depression among people with active IBD.

Exeter hospital between January and June 2017. Participants were excluded if they were too physically unwell, if they suffered from severe mental disorder, including severe depression, significant suicidal risk or active psychosis.

\subsection{1 | Sample size calculation}

Making the a priori assumption that key variables of interest would be normally distributed, we calculated that a sample of 85 subjects would provide $\geq 80 \%$ power to detect bivariate correlations of at least $r=0.3$ between measures of emotional processing, markers of disease severity and depression at the $5 \%$ level of significance (2-sided). Also, we expected this number of subjects would provide sufficient power to conduct multivariable regression analyses using up to 8 independent variables, based on the rule-of-thumb of 10 participant per independent variable added. ${ }^{33}$

\section{2 | Baseline assessments}

Data were obtained using a combination of self-report questionnaires, computerized assessment and by extraction of relevant clinical information from medical records.

\subsection{1 | Questionnaire assessments}

A purpose-designed questionnaire was used to record sociodemographic characteristics including: age, sex, relationship status (categorized as "In relationship" versus "Other"), educational status (years of education) and employment status (categorized as "In employment" versus "Other"), smoking status (current smoker, ex-smoker, never smoked) and previous treatments for depression.

We used the following validated questionnaire assessments:

The frequency of depressive symptoms in the previous 2 weeks was assessed using 9-item Patient Health Questionnaire, PHQ-9. ${ }^{34}$ Scores could range from 0 to 27 , with higher scores indicating worse depression. A cut-off score of $\geq 10$ indicates moderate depressive 
symptoms and we used this cut-off to identify cases of depression among our participants.

Anxiety was measured using the 7-item General Anxiety Disorder Assessment, GAD-7, ${ }^{35}$ perceived social support was assessed using the seven item ENRICHD social support inventory ${ }^{36-38}$ and recent life stresses were assessed using the 12-item List of Threatening Experiences questionnaire. ${ }^{39}$ The EQ-5D questionnaire was used to assess generic health-related quality of life ${ }^{40}$ and the 10 -item, Short Inflammatory Bowel Disease questionnaire was used to record disease-specific health-related quality of life. ${ }^{41,42}$

Questionnaire assessments were completed in clinic following recruitment, though participants could take them home to complete, if preferred.

\subsection{2 | Data extracted from medical records}

We recorded demographic data, smoking status, age at diagnosis, disease duration, Montreal Classification, ${ }^{43}$ prior medical and drug history and previous IBD. Patients postcodes were used to identify the degree of social deprivation, as determined using the index of multiple deprivation (IMD). ${ }^{44}$

Inflammatory bowel disease activity at the time of recruitment was categorized as active versus inactive via retrospective inspection of medical records. Two experienced clinicians (JG, NH) independently reviewed clinical and laboratory information for each participant at the time of recruitment to the study, blind to the outcomes of any research assessments. Disagreements in ratings were resolved though consensus, with referral to a third independent clinician (NAK) if agreement could not be reached.

\subsection{3 | Computerized assessment of emotional processing}

We selected specific tests from a validated computerized neuropsychological test battery (EMOTICOM) ${ }^{45}$ to assess performance on emotional perception, emotional memory and reinforcement learning, which we recently showed were aspects of social and emotional processing most likely to be influenced by inflammation. ${ }^{30}$ All tasks were presented on a Hewlett Packard 755 laptop computer with 15.6" touchscreen.

\subsubsection{Emotional recognition task}

The Emotional Recognition Task (ERT) assessed an individual's ability to recognize basic emotions (happy, sad, angry and fearful) from 80 images of people's eyes (20 of each emotion), with 10 levels of intensity for each emotion. In each trial a fixation cross was presented in the center of the screen (random duration between 1500 and 2500 milliseconds), followed by an image of eyes (250 milliseconds). The image was immediately replaced by a grey mask (150 milliseconds), following which the participant made a forced choice from four emotions (happy, sad, angry or fearful). There were in addition 16 filler trials in which participants were asked to select the age of the eyes in the image (child, young adult, middle-aged adult and older adult). Performance on the emotional recognition task is reported as "emotional recognition bias", calculated as the percentage accuracy for recognition of happy expressions minus the percentage accuracy for recognition of sad expressions.

\subsection{5 | Emotional memory recognition task}

The Emotional Memory Recognition Task (EMRT) was presented in two parts. During the first phase (encoding) participants were shown 30 photographic scenes without people (10 positive, 10 negative and 10 neutral). In each trial a fixation cross was displayed in the centre of the screen for 1000 milliseconds, followed by an image also displayed for 1000 milliseconds. Participants were asked to make ratings of valence ( 1 = negative, 9 = positive) and intensity ( 1 = not at all, 9 = extremely) for each image. In the second phase (retrieval) conducted 30 minutes later, participants were shown 30 images from the encoding phase, each paired with new photographs, which were mirror images of those seen during encoding. Participants were asked to identify the image seen during encoding. Performance on the emotional memory task is reported as "emotional memory bias", calculated as the percentage accuracy recall of positive scenes minus the percentage accuracy for recall of negative scenes.

\subsection{6 | Reinforcement learning}

The Reinforcement Learning Task (RLT) assessed speed of learning of visual patterns associated with reward (winning points) and punishment (losing points). Participants were shown pairs of colored circles and were instructed to select one of the circles which they thought would be most likely to win money. Participants were expected to learn through sampling the circles which of the two circles was most likely to deliver a win, with probabilities set at $70 / 30 \%$, unknown to participants. Feedback was given after each selection and a cumulative tally was displayed. The task was presented in two parts. First, there were 120 trials in the learning phase. In each trial a fixation cross was presented (random duration between 500 to 1500 milliseconds) followed by 1 of 4 possible pairs of colored circles. The circles remained until the participant selected one circle, after which feedback was displayed for 1000 milliseconds. There were two conditions: reward ( 2 pairs/60 trials) or punishment (2 pairs/60 trials). In the reward condition feedback consists of a win (win 50p) or failure to win (win 0p), and in the punishment condition feedback consists of a loss (lose 50p) or avoidance of loss (lose 0p). Next, in the transfer phase there were 48 trials where all possible pairs of circles were presented. Participants were instructed to continue to select their preferred circle, although no feedback was provided in this phase.

Performance on the RLT is reported using learning rate (ie, how fast the participant learned new information related with winning and losing, where high scores show that learning was more rapid), calculated from the learning phase only (not the transfer phase) and 
the performance temperature (a measure of the randomness in responding). On initial inspection of the learning data, it became clear that some subjects were performing no better than chance (ie, there was no evidence of learning, with performance on the task at or below $50 \%$ correct), which resulted in poor model fit. Once we had excluded these non-performers, the model that accounted best for the participant's performance was the reinforcement learning model with separate parameters for rewards and losses. Thus, results for reinforcement learning data presented below are limited to individuals showing evidence of learning on the task.

\subsection{7 | Blood samples}

Blood was collected in $7.5 \mathrm{~mL}$ EDTA tubes and centrifuged at $2500 \mathrm{~g}$ for 10 minutes at $4 \mathrm{C}$ in a Thermo Scientific Heraeus 16R Megafuge. Within 30 minutes of venipuncture the separated plasma was divided into 3 aliquots (minimum $0.5 \mathrm{~mL}$ per aliquot) and then frozen at $-80^{\circ} \mathrm{C}$ for subsequent assay for C-reactive protein (high sensitivity assay, hs-CRP).

\subsection{8 | Hs-CRP assay}

Hs-CRP levels were established using Cardiac C-reactive protein (latex) high sensitive, particle enhanced immunoturbidimetric assay on the 702 module of a Roche/Hitachi cobas 8000 automated analyzer. The lower detection limit for hs-CRP using this system was $0.15 \mathrm{mg} / \mathrm{L}$. One subject had levels below this lower limit of detection ( $<0.15 \mathrm{mg} / \mathrm{L}$ ) and, for the purposes of analysis hs-CRP as a continuous variable, levels for this individual were assumed to equal $0.15 \mathrm{mg} / \mathrm{L}$. In addition, hs-CRP levels were also divided into low and high hs-CRP categories $(\leq 3 \mathrm{mg} / \mathrm{L}$ and $>3 \mathrm{mg} / \mathrm{L}$, respectively).

\section{3 | Statistical considerations}

Preliminary examination of the continuous variables using 1-sample Kolmogorov-Smirnov tests revealed that our a priori assumption that key variables would be normally distributed was incorrect. In fact, the vast majority of variables were non-parametrically distributed. Standard transformations did not increase normality, so non-parametric statistical techniques were used throughout. Sociodemographic, IBD and psychological characteristics are summarized using median and interquartile range, or number and percentages, as appropriate. Differences in sociodemographic, IBD and psychological variables according to depression status were examined using the Mann Whitney $U$ test for continuous data. Chi-square tests were used to compare categorical data, with Fisher's Exact test used where contingency tables included cells with expected frequencies $<5$.

To identify variables independently associated with depression, multivariable logistic regression analysis was conducted that included the following independent variables: Block 1: age, sex socioeconomic status, social support, Block 2: IBD type (Crohn's
Disease, Ulcerative Colitis, Unclassified) and IBD activity (Active vs Inactive IBD), Block 3: psychological characteristics (bias in emotional processing). Due to the highly non-parametric distribution of independent variables, for the purposes of the regression analyses continuous independent variables were converted to binary categories, using a median split unless other established cut-offs were more appropriate (ie PHQ-9 $\geq 10$ and hs-CRP > $3 \mathrm{mg} / \mathrm{L}$ ).

We explored the role of emotional recognition bias as a potential mediator of the association of disease activity with depression using a causal steps approach, based the methods of Baron and Kenny. ${ }^{46}$ A series of 3 logistic regression analyses were conducted: (a) Depression regressed on disease activity, (b) Emotional recognition bias regressed on disease activity, and (c) Depression regressed on both disease activity and emotional recognition bias, in the same model.

Mediation was considered to have occurred if all of the following conditions were met (see Figure 1 for illustration):

1. Disease activity predicted depression (the total, unadjusted, effect of predictor on outcome, path c').

2. Disease activity predicted emotional recognition bias (the direct effect of predictor on mediator, path a).

3. Emotional recognition bias significantly predicted depression in a model that also included disease activity (path $b$, the direct effect of mediator on outcome).

4. The regression coefficient of disease activity on depression in the model that also included emotional recognition bias (path $c$, the direct effect of predictor on outcome) was smaller than the coefficient of the total effect (path c').

If the causal steps approach indicated findings consistent with mediation, a bootstrapping method with 5000 samples and bias corrected confidence intervals was used to determine significance of the mediated effect. ${ }^{47}$

\subsection{Ethical statement}

All participants provided full informed consent. Full ethical permission was granted by South West - Cornwall and Plymouth research ethics committee, reference: 16/SW/0209.

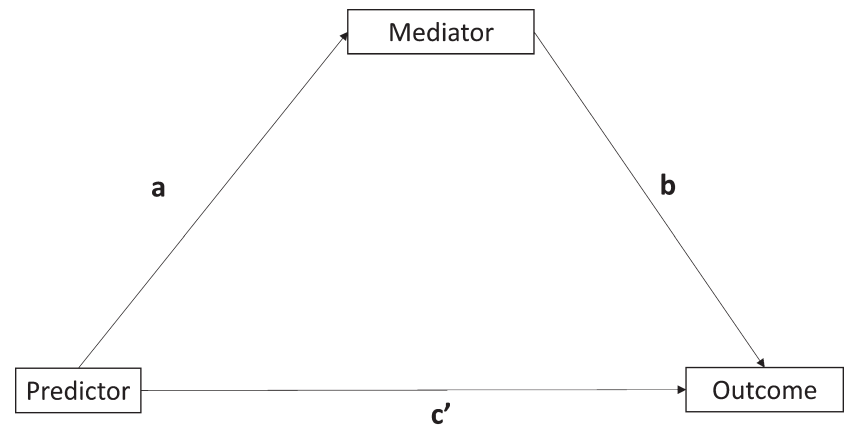

(c)

FIGURE 1 Method used for testing mediation 


\section{3 | RESULTS}

\section{1 | Participant characteristics}

One hundred and twenty patient participants agreed to participate in the study. Sixty-eight patients (57\%) had Crohn's disease, 49 (41\%) had ulcerative colitis and the remaining $3(2 \%)$ had IBD unclassified. The median duration of IBD was 9.2 years (IQR: 4.2-15.2), with the median age of onset being 29.9 years (IQR: 22.3-43.6). Forty-six patients (38\%) were taking anti-TNF drugs to control their IBD. Full baseline characteristics of study participants can be seen in Table 1.

Of the 120 patients recruited, 35 (29\%) were classified as having active IBD. Those with active disease had higher hs-CRP levels (median levels $5.0 \mathrm{mg} / \mathrm{L}$ [IQR: 2.75-9.38] vs $1.2 \mathrm{mg} / \mathrm{L}$ [IQR: 0.50-2.70], Mann Whitney, $P<0.0005)$ and higher white cell counts (median $8.4 \times 10^{9} /$ L [IQR: 6.80-9.80] vs $6.6 \times 10^{9} /$ L [IQR: $\left.5.55-7.80\right]$, Mann Whitney, $P<0.0005)$. Furthermore, those with active disease were more likely to be taking corticosteroids ( $20 \%$ vs $1.2 \%, P=0.001)$ and less likely to be taking anti-TNF drugs ( $20 \%$ vs $45.9 \%, P=0.008)$. Participants with active disease had worse generic and disease specific health-related quality of life (EQ-5D index value and VAS; SIBDQ Systemic, Social, Bowel and Emotional domains of the Short IBD questionnaire, all $P^{\prime} \mathrm{s} \leq 0.005$ ).

Overall participants showed a positive bias in emotion recognition [median emotional recognition bias $=+15 \%$ (IQR: 0.0-30.0)] and a negative bias in emotional memory [median emotional memory bias $=-10 \%(I Q R=-30.0$ to 0.0$)$ ]. Emotional recognition bias was less positive in people with active disease [median recognition bias $+5 \%$ (IQR: -5.0 to 20.0 ) vs $+15 \%$ (IQR: $2.50-35.0$ ), Mann Whitney, $P=0.028$ ], but was not significantly associated with hs-CRP (Spearman's correlation coefficient $(\rho)=-0.04$, $\mathrm{n}=101, \mathrm{P}=0.73)$ or white cell count $(\rho=-0.01, \mathrm{n}=112, \mathrm{P}=0.91)$. Emotional memory bias and learning rate (win or loss) were not significantly associated with disease activity or markers of inflammatory activity.

\subsection{Sociodemographic, IBD and psychological factors associated with depression}

Of the 120 participants recruited, 105 returned questionnaires, of which 104 included completed depression assessments. There were no significant differences with regards to age, sex, socioeconomic status or disease activity between those 104 returning the depression assessment and the 16 who did not.

Twenty-six participants (25\%) were depressed ( $\mathrm{PHQ}-9$ score $\geq 10$ ). Sociodemographic, clinical and psychological factors that showed univariate associations with depression can be seen in Table 2. Of note, those with depression were significantly more likely to be female, lack social support, have active IBD, not be taking anti-TNF alpha inhibitors, have worse quality of life and exhibit less positive bias on the emotional recognition task [median emotional recognition bias $=+2.5$ (IQR: -25.0 to 15.0) in depressed vs $+15 \%(0.0-35.0)$ in the non-depressed, Mann-Whitney, $P=0.002]$. Depression was not associated with laboratory markers of inflammatory activity (hsCRP or white cell count), emotional memory, reinforcement learning related to reward or loss.

Using multivariable logistic regression, the overall model was significant (Chi-square $=24.9, P=0.001$, Cox and Snell Rsquare $=0.22$. Within the model, depression was independently associated with less social support [odds ratio $(O R)=0.25(95 \%$ $\mathrm{Cl}=0.08-0.76), \mathrm{P}=0.02]$ and greater disease activity $[\mathrm{OR}=3.6$ (95\% Cl $=1.14-11.60) P=0.03$ ) (Table 3). Age, sex, IMD and emotional recognition bias $[\mathrm{OR}=0.39,(95 \% \mathrm{Cl}=0.12-1.27), P=0.12$ ] did not make any significant independent contribution to the full regression model.

Since disease activity and emotional recognition bias showed a significant univariate association with each other, we explored the effect of removing disease activity from the regression model. When disease activity was removed from the model, the overall model remained significant (Chi-square $=20.1, P=0.003$, Cox and Snell R-square $=0.182$ ), and less positive emotional recognition bias $(B=-1.20, S E=0.58, P=0.04, \operatorname{Exp}(B)=0.30)$ and less social support $(B=-1.31, S E=0.55, P=0.02, \operatorname{Exp}(B)=0.27)$ were the only variables to make a significant independent contribution to the model.

Using the causal steps approach, disease activity was associated with emotional recognition bias $(B=-0.93, P=0.043)$ and both disease activity $(B=1.47, P=0.003)$ and emotional recognition bias $(B=-1.27, P=0.019)$ predicted depression. The contribution of disease activity to the model decreased when emotional recognition was added to the model $(B=1.29, P=0.012$, Figure 2$)$, consistent with emotional recognition partially mediating the effects of disease activity on depression (Figure 1B). Bootstrap test of indirect effect was significant, and proportion of total effect mediated $=19.8 \%$. Disease activity was also associated with anxiety $(B=1.2, P=0.03)$, though emotional recognition bias did not meet criteria for mediation in this association, since the association between emotional recognition bias and anxiety was non-significant (B $=-0.48, P=0.38)$.

\section{4 | DISCUSSION}

We found that depression affected $25 \%$ of people with IBD and was associated with a wide range of sociodemographic, IBD-related and psychological factors including less positive biases in emotional recognition. However, on multivariable analysis, depression was predicted by a lack of social support and greater IBD activity, only. Causal steps analysis suggested that emotional recognition bias partially mediated the relationship between disease activity and depression, as we hypothesized.

This is the first study to explore links between disease activity and emotional processing biases, with the aim of understanding mechanisms underpinning the development of depression among people with IBD. Strengths of our study include the recruitment of a representative sample of outpatients with IBD and the use of 
TAB LE 1 Characteristics of subjects recruited (Median (IQR) or $n(\%))$

\begin{tabular}{|c|c|c|}
\hline \multicolumn{3}{|l|}{ Subject characteristic } \\
\hline \multicolumn{3}{|c|}{ Socio-demographic characteristics } \\
\hline Age & Years & $44.0(33.3-56.0)$ \\
\hline Sex & Male & $52(43.3)$ \\
\hline Ethnicity & White British & $120(100)$ \\
\hline Socio-economic & IMD decile & $6.0(4.0-8.0)$ \\
\hline Education $(n=98)$ & Years & $15.0(12.0-18.0)$ \\
\hline Employment & Working & $68(64.8)$ \\
\hline \multirow[t]{3}{*}{ Smoking } & Current & $10(8.3)$ \\
\hline & Ex & $21(17.5)$ \\
\hline & Never & $89(82.5)$ \\
\hline Relationships & In a relationship & $73(69.5)$ \\
\hline Lives alone & & $14(13.3)$ \\
\hline ENRICHD $(n=105)$ & & $26.0(22.0-29.0)$ \\
\hline \multicolumn{3}{|l|}{ IBD characteristics } \\
\hline \multirow[t]{3}{*}{ Disease type } & Crohn's & $68(56.7)$ \\
\hline & UC & $49(40.8)$ \\
\hline & IBD-U & $3(2.5)$ \\
\hline Disease duration & Years & $9.2(4.2-15.2)$ \\
\hline Age at diagnosis & Years & $29.9(22.3-43.6)$ \\
\hline Disease activity & Active disease & $35(29.2)$ \\
\hline \multicolumn{3}{|c|}{ Crohn's disease montreal classification $(n=68)$} \\
\hline \multirow[t]{3}{*}{ Age } & A1: Age $<17$ & 9 (13.2) \\
\hline & A2: $17-40$ & $42(61.8)$ \\
\hline & A3: $>40$ & $17(25.0)$ \\
\hline \multirow[t]{4}{*}{ Location of Crohn's } & L1: Ileal & $28(41.2)$ \\
\hline & L2: Colonic & $17(25.0)$ \\
\hline & L3: Ileocolonic & $23(33.8)$ \\
\hline & + L4: Upper GI & $12(17.6)$ \\
\hline \multirow[t]{4}{*}{ Crohn's behaviour } & B1: Inflammatory & $41(60.3)$ \\
\hline & B2: Stricturing & $21(30.9)$ \\
\hline & B3: Penetrating & $6(8.8)$ \\
\hline & $+\mathrm{p}$ : Perianal & $10(14.7)$ \\
\hline \multirow{3}{*}{$\begin{array}{l}\text { UC montreal } \\
\text { classification }\end{array}$} & E1: Proctitis & $8(15.4)$ \\
\hline & E2: Distal colitis & $20(38.5)$ \\
\hline & E3: Total colitis & $24(46.2)$ \\
\hline \multirow[t]{7}{*}{ Medications } & 5 ASA & $32(26.7)$ \\
\hline & Corticosteroids & $8(6.7)$ \\
\hline & Thiopurine & $46(38.3)$ \\
\hline & Methotrexate & $4(3.3)$ \\
\hline & Anti-TNF & $46(38.3)$ \\
\hline & Vedolizumab & $22(18.3)$ \\
\hline & Ustekinumab & $2(1.7)$ \\
\hline
\end{tabular}

TABLE 1 (Continued)

\begin{tabular}{|c|c|c|}
\hline \multicolumn{3}{|l|}{ Subject characteristic } \\
\hline \multirow[t]{5}{*}{ Prior surgeries } & None & $94(78.3)$ \\
\hline & Ileocecal resection & $20(16.7)$ \\
\hline & Subtotal colectomy & $3(2.5)$ \\
\hline & $\begin{array}{l}\text { Small bowel } \\
\text { resection }\end{array}$ & $2(1.7)$ \\
\hline & $\begin{array}{l}\text { Right } \\
\text { hemicolectomy }\end{array}$ & $1(0.8)$ \\
\hline \multirow[t]{3}{*}{$\begin{array}{l}\text { Baseline laboratory } \\
\text { indices }\end{array}$} & Haemoglobin (g/L) & $\begin{array}{l}134.0 \\
(124.0-141.8)\end{array}$ \\
\hline & $\mathrm{MCV}(\mathrm{fL})$ & $89.3(85.6-94.0)$ \\
\hline & $\begin{array}{l}\text { White cell count } \\
\left(10^{9} / \mathrm{L}\right)\end{array}$ & $6.9(5.8-8.6)$ \\
\hline \multirow[t]{4}{*}{$\begin{array}{l}\text { Baseline laboratory } \\
\text { indices }\end{array}$} & $\begin{array}{l}\text { Platelet count } \\
\left(10^{9} / \mathrm{L}\right)\end{array}$ & $\begin{array}{l}242.0 \\
(212.3-304.8)\end{array}$ \\
\hline & Haematocrit (vol\%) & $39(37-41)$ \\
\hline & $\begin{array}{l}\text { Hs-CRP } \\
(\mathrm{n}=107 \mathrm{mg} / \mathrm{L})\end{array}$ & $1.7(0.80-4.70)$ \\
\hline & $\begin{array}{l}\text { Hs C-reactive } \\
\text { protein }>3 \mathrm{mg} / \mathrm{L} \\
(\mathrm{n}=107)\end{array}$ & $40(37.4)$ \\
\hline \multirow[t]{4}{*}{ SIBDQ subscales } & Systemic $(n=105)$ & $4.5(3.3-5.5)$ \\
\hline & Social $(n=104)$ & $6.0(5.0-7.0)$ \\
\hline & Bowel $(n=104)$ & $5.3(4.3-6.0)$ \\
\hline & Emotional $(n=104)$ & $5.0(3.7-6.0)$ \\
\hline Total SIBDQ & $(n=105)$ & $4.9(4.3-5.8)$ \\
\hline EQ-5D VAS & $(n=105)$ & $75.0(62.5-85.0)$ \\
\hline EQ-5D index value & $(n=105)$ & $0.70(0.72-0.95)$ \\
\hline \multicolumn{3}{|c|}{ Psychological characteristics } \\
\hline PHQ-9 & $(n=104)$ & $5.5(3.0-10.5)$ \\
\hline PHQ $-9 \geq 10$ & $(n=104)$ & $26(25.0)$ \\
\hline GAD-7 & $(n=105)$ & $5.0(1.0-8.0)$ \\
\hline GAD-7 $\geq 10$ & $(n=105)$ & 18 (17.1) \\
\hline Recent Life stresses & Yes & $60(57.1)$ \\
\hline \multicolumn{3}{|l|}{ Cognitive assessments } \\
\hline $\begin{array}{l}\text { Emotional recogni- } \\
\text { tion bias }(n=112)\end{array}$ & & $15.0(0.0-30.0)$ \\
\hline $\begin{array}{l}\text { Emotional memory } \\
\text { bias }(n=108)\end{array}$ & & $-10(-30$ to 0.0$)$ \\
\hline \multirow[t]{4}{*}{$\begin{array}{l}\text { Reward and punish- } \\
\text { ment processing }\end{array}$} & $\begin{array}{l}\text { Learning rate Win } \\
\left(\mathrm{n}=48^{\mathrm{a}}\right)\end{array}$ & $0.10(0.02-0.56)$ \\
\hline & $\begin{array}{l}\text { Temperature Win } \\
(\mathrm{n}=48)\end{array}$ & $0.17(0.03-0.46)$ \\
\hline & $\begin{array}{l}\text { Learning rate Loss } \\
\left(\mathrm{n}=48^{\mathrm{a}}\right)\end{array}$ & $0.22(0.06-0.53)$ \\
\hline & $\begin{array}{l}\text { Temperature loss } \\
(\mathrm{n}=48)\end{array}$ & $1.0(0.72-1.08)$ \\
\hline
\end{tabular}

Note: Number of participants $(n)=120$, unless otherwise stated. Abbreviations: IMD: Index of multiple deprivation; IQR: interquartile range; SIBDQ: Short Inflammatory Bowel Disease questionnaire. andividuals showing no evidence of learning were excluded from these results. 
TABLE 2 Comparing depressed with non-depressed

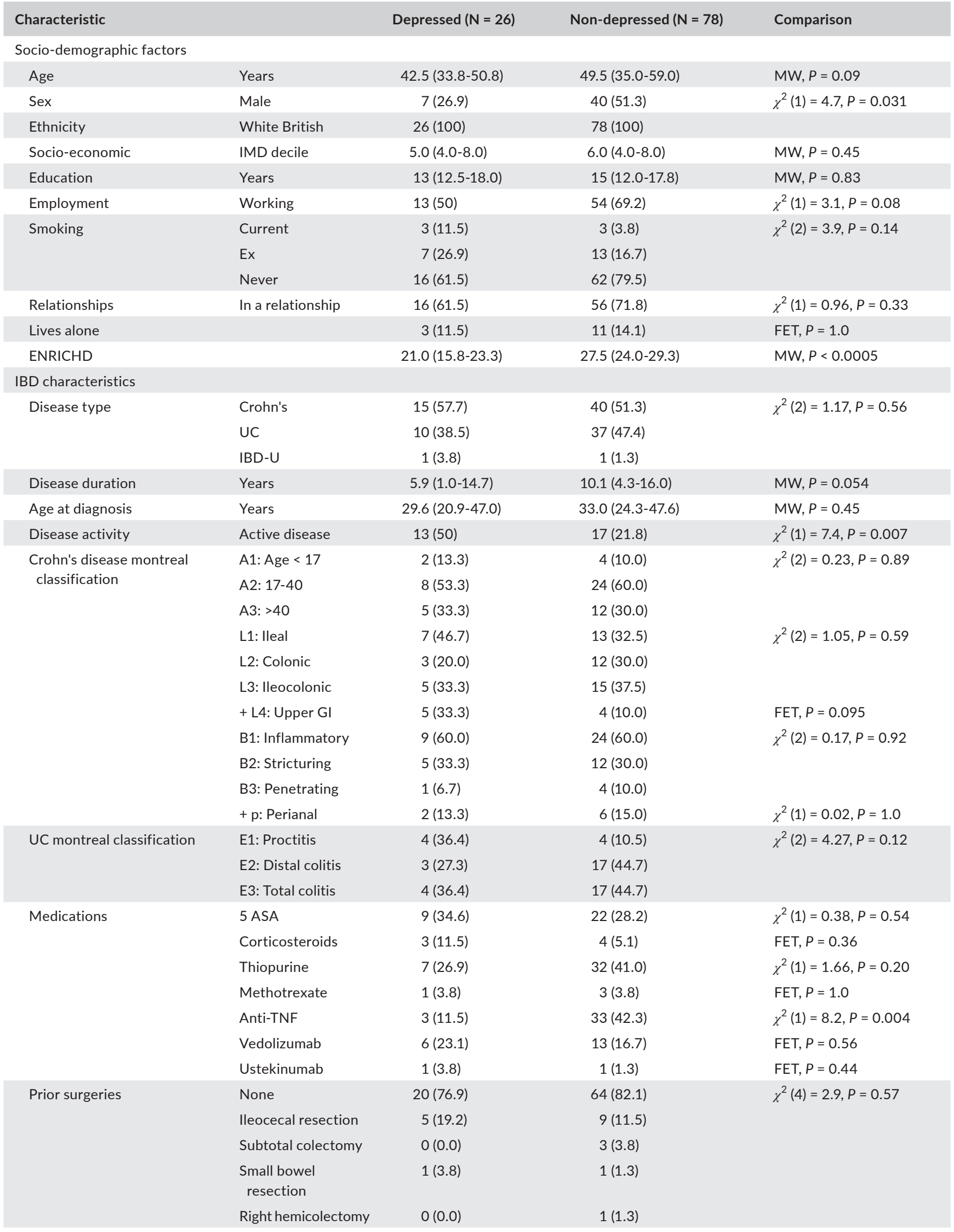




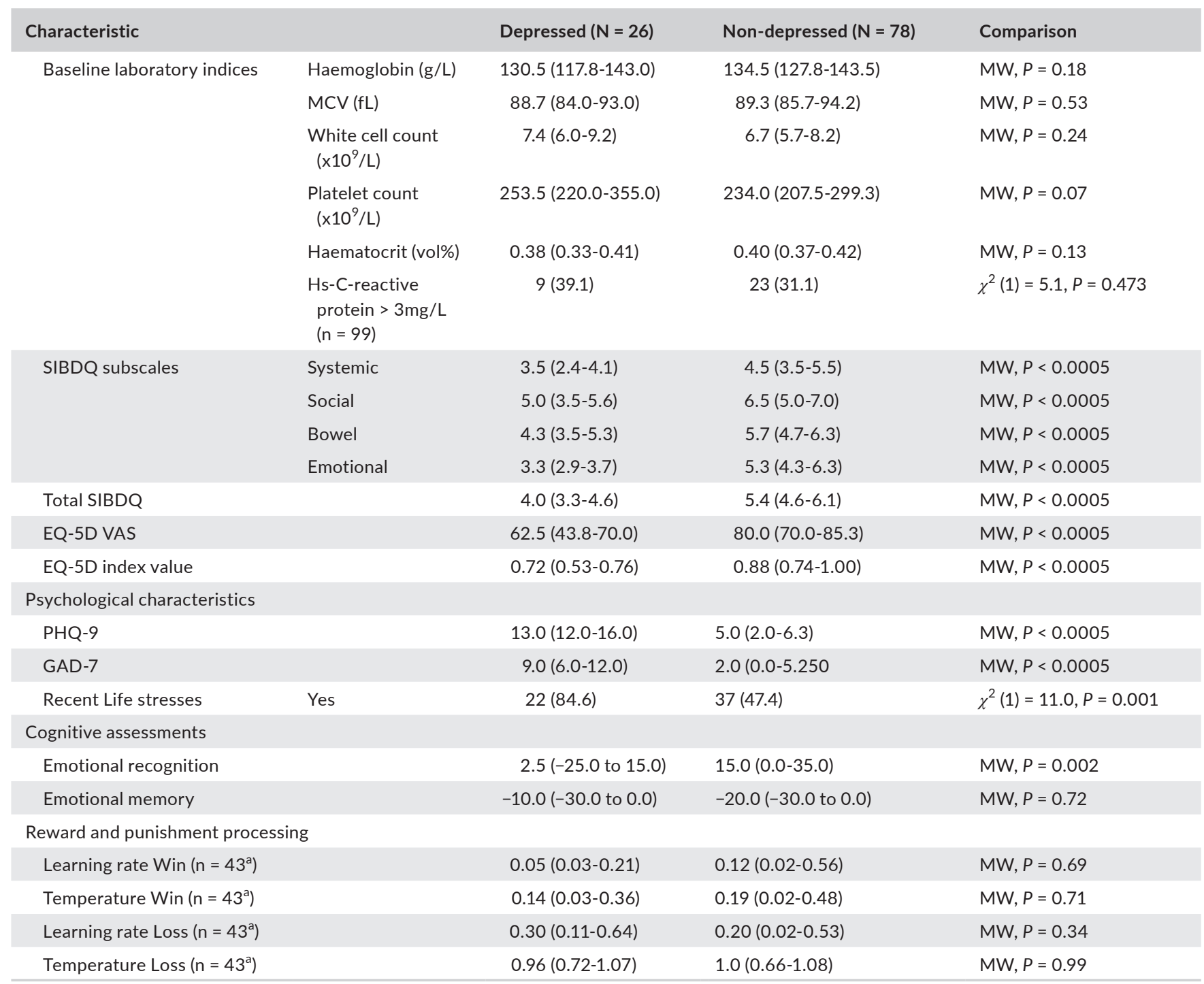

Note: Number of participants $(n)=104$ (the number completing the depression assessment), unless otherwise stated; FET, Fishers Exact Test (2sided), used when cross-tabulation includes cells with expected count $<5$.

Abbreviations: IMD: Index of multiple deprivation; IQR: interquartile range; SIBDQ: Short Inflammatory Bowel Disease questionnaire.

andividuals showing no evidence of learning were excluded from these results.

standardized assessments to record key variables of interest, so we are confident that our findings are generalizable, valid and reliable. Finally, our measures of emotional processing were selected from a battery of tests designed specifically to evaluate changes in emotional processing associated with mental disorders, informed by a systematic review of experimental findings relating to acute inflammation.

The main weakness of our study was its cross-sectional design, meaning that we could not determine the direction of causation of any of the observed associations. Despite our causal steps approach, we recognize that mediation analyses based on cross-sectional data must be regarded as preliminary, since spurious and inflated associations may occur. ${ }^{48}$ Also, since conventional symptom scores are heavily weighted by quality of life and well-being domains that can be influenced directly by depression, use of such scores to assess
IBD activity risks inflating the apparent association between IBD activity and depression. To avoid this, we used the opinions of expert gastroenterologists to determine clinical disease activity via retrospective inspection of medical records, blinded to depression status and the outcomes of research assessments. Whilst the fact that people whose IBD was classified as active had significantly higher hsCRP levels and worse health-related quality of life scores, provides some confirmation of the validity of our IBD activity assessment method, we acknowledge that such an assessment is fundamentally subjective and therefore vulnerable to bias.Future studies should consider using more valid and reliable measures of IBD activity such as fecal calprotectin.

We interpret our findings as confirming that depression is common in hospital outpatients with IBD, and that having active IBD and lacking of social support were the strongest predictors of 
TABLE 3 Multivariable predictors of depression

\begin{tabular}{|llll|}
\hline & OR & $95 \% \mathrm{Cl}$ & Sig. \\
\hline Age $<45$ y vs $\geq 45$ y & 0.71 & $0.23-2.24$ & 0.56 \\
\hline Sex & 2.11 & $0.68-6.56$ & 0.20 \\
\hline IMD category (high vs low) & 0.82 & $0.27-2.51$ & 0.72 \\
\hline Social support (high versus low) & 0.25 & $0.08-0.76$ & 0.02 \\
\hline Type of IBD (Crohn's vs UC) & 1.44 & $0.54-3.88$ & 0.47 \\
\hline Disease activity (Active vs inactive) & 3.64 & $1.14-11.60$ & 0.03 \\
\hline $\begin{array}{c}\text { Emotional Recognition categorical } \\
\text { (more positive vs more negative) }\end{array}$ & 0.39 & $0.12-1.27$ & 0.12 \\
\hline Constant & 0.18 & & \\
\hline
\end{tabular}

Abbreviations: $\mathrm{Cl}$ : confidence intervals; IMD: Index of multiple deprivation; OR: Odds ratio.

depression. This finding is consistent with our research in rheumatoid arthritis, which showed that depression was more likely among people who experienced life difficulties in both disease-related and non-disease related domains. ${ }^{49}$ Due to our small population size and the loss of statistical power due to shifting from the planned multivariable linear regression to logistic regression to accommodate the non-parametric distribution of our key variables, we cannot conclude that other factors are unimportant in contributing to depression at an individual level, merely that disease activity and social support were important predictors of depression among our participants.

Whilst depression was associated with clinical disease activity, we did not find that depression was associated with hs- C-reactive protein. This would seem to contradict the ever growing observational evidence that depression is associated with inflammation. One explanation could be that most patients recruited to this study were taking drugs that are recognized to reduce inflammation, such as corticosteroids and TNF-alpha inhibitors, which could have moderated the association between inflammation and depression. ${ }^{50-52}$ Common use of such powerful anti-inflammatory drugs in clinical populations could mean that findings from research into acute inflammation in healthy individuals performed in laboratory settings or using population-based observational studies cannot necessarily be extrapolated directly to clinical

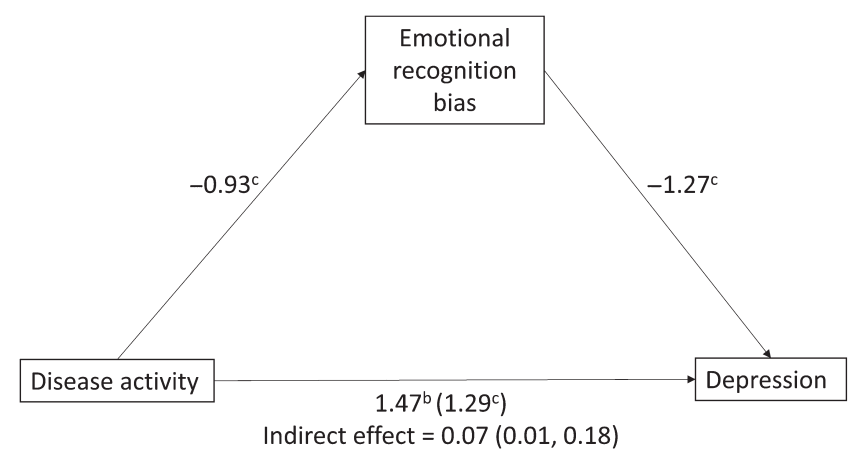

FIGURE 2 Path diagram of mediation. Paths annotated with unstandardized regression coefficients. ${ }^{\mathrm{a}} P \leq 0.001,{ }^{\mathrm{b}} P \leq 0.01$, ${ }^{c} P \leq 0.05$ populations receiving such treatments. Another explanation could be that we did not measure mediators of inflammation sufficiently thoroughly, being limited to CRP, an inactive marker of inflammation. Furthermore, exclusion of IBD sufferers with most severe depression and most severe IBD may have weakened associations that would have otherwise been apparent if people with more severe health problems had been included. Finally, of course, this lack of association could indicate that there is no association between inflammation and depression among people with IBD.

Our finding of a reduction of positive bias during emotional recognition in people with active compared to inactive IBD was robust and consistent with the previous small fMRI study of patients with ulcerative colitis. ${ }^{53}$ Our finding that less positive biases in emotional recognition partially mediated the association between IBD activity and depression is new and starts to elucidate the mechanisms underpinning depression among people with IBD, and possibly other long term conditions.

Further research is required to investigate mechanisms underlying the development and maintenance of depression and, in particular, to test our hypotheses that that the association between disease activity/inflammation and depression might be mediated via emotional processing biases. Larger participant numbers will increase statistical power so possibly identifying other factors that are associated with depression but also facilitate analysis on subgroups not taking antiinflammatory drugs, which may influence the associations between depression and disease activity. Study of populations with other chronic inflammatory conditions may reveal subtle differences in the effects of inflammation and anti-inflammatory drugs on depression. Assessment of cytokines and a broader range of cognitive processes may provide a more comprehensive investigation of mechanisms underlying depression. Prospective study design will enable stronger causal inferences to be drawn if the nature of the temporal relationships between presumed predictors and dependent variables can be established. Emotional recognition biases did not mediate the association between disease activity and anxiety in this preliminary study, though anxiety should be considered alongside depression in future studies of the impact of inflammation. Our findings raise the possibility that psychological interventions targeting emotional recognition biases among people with IBD, could be used to treat or even prevent depression in high risk individuals, such as those with active IBD, and thereby possibly improve medical as well as psychological outcomes.

\section{CONFLICT OF INTEREST}

None of the authors have conflicts of interest that relate directly to the submitted work. For transparency, the author declare the following potential conflicts that are unrelated to the current work: Goodhand has received honoraria from Falk, Abbvie and Shield Therapeutics; grant funding from Pharmacosmos (co-app); support from the Royal Devon and Exeter Externally Funded Research (EFR) scheme. Kennedy has received: grants from International Serious Adverse Events Consortium and Pharmacosmos; personal fees from Falk, Allergan, Takeda and Pharmacosmos. Ahmad has received: 
honoraria from Celltrion, NAPP, MSD, Abvie, Pfizer, Takeda, Janssen and Immunodiagnostik; research grants from Celltrion, NAPP, MSD, Abvie, Pfizer, Tillots and Immunodiagnostik; education grants/travel grants or fellowship from NAPP, MSD, Abvie, Takeda and Tillots; Equipment grants from Immunodiagnostik; sponsorship of post doc within department from Immunodiagnostik. Dickens has received research funding (co-app) from Pharmacosmos.

\section{AUTHOR CONTRIBUTIONS}

Wilkinson: contributed to design, conducted data collection, conducted initial analyses, wrote first draft and provided final approval. Dickens: conceived the original idea, provided the initial design and provided overall supervision for data collection, analyses, interpretation, draft writing and final approval. Goodhand, Kennedy, Ahmad, Trick, Knight and Heerasing contributed to: design, data collection, interpretation, draft writing and final approval. Bland, Elliott, Valton Roiser contributed to: analyses, interpretation, draft writing and final approval.

\section{ORCID}

Chris Dickens iD https://orcid.org/0000-0001-6326-7544

\section{REFERENCES}

1. Fuller-Thomson E, Sulman J. Depression and inflammatory bowel disease: findings from two nationally representative Canadian surveys. Inflamm Bowel Dis. 2006;12(8):697-707.

2. Roman AL, Munoz F. Comorbidity in inflammatory bowel disease. World J Gastroenterol. 2011;17(22):2723-2733.

3. Walker JR, Ediger JP, Graff LA, et al. The Manitoba IBD cohort study: a population-based study of the prevalence of lifetime and 12-month anxiety and mood disorders. Am J Gastroenterol. 2008;103(8):1989-1997.

4. Walker EA, Gelfand MD, Gelfand AN, Creed F, Katon WJ. The relationship of current psychiatric disorder to functional disability and distress in patients with inflammatory bowel disease. Gen Hosp Psychiatry. 1996;18(4):220-229.

5. Vidal À, Gómez-Gil E, Sans M, et al. Health-related quality of life in inflammatory bowel disease patients: the role of psychopathology and personality. Inflamm Bowel Dis. 2008;14(7):977-983.

6. Hyphantis TN, Tomenson B, Bai M, Tsianos E, Mavreas V, Creed F. Psychological distress, somatization, and defense mechanisms associated with quality of life in inflammatory bowel disease patients. Dig Dis Sci. 2010;55(3):724-732.

7. Guthrie E, Jackson J, Shaffer J, Thompson D, Tomenson B, Creed F. Psychological disorder and severity of inflammatory bowel disease predict health-related quality of life in ulcerative colitis and Crohn's disease. Am J Gastroenterol. 2002;97(8):1994-1999.

8. Zhang CK, Hewett J, Hemming J, et al. The influence of depression on quality of life in patients with inflammatory bowel disease. Inflamm Bowel Dis. 2013;19(8):1732-1739.

9. Farrokhyar F, Marshall JK, Easterbrook B, Irvine EJ. Functional gastrointestinal disorders and mood disorders in patients with inactive inflammatory bowel disease: prevalence and impact on health. Inflamm Bowel Dis. 2006;12(1):38-46.
10. de Boer AG, Sprangers MA, Bartelsman JF, de Haes HC. Predictors of health care utilization in patients with inflammatory bowel disease: a longitudinal study. Eur J Gastroenterol Hepatol. 1998;10(9):783-789.

11. Drossman DA, Leserman J, Mitchell CM, Li ZM, Zagami EA, Patrick DL. Health status and health care use in persons with inflammatory bowel disease. A national sample. Dig Dis Sci. 1991;36(12):1746-1755.

12. Mittermaier C, Dejaco C, Waldhoer T, et al. Impact of depressive mood on relapse in patients with inflammatory bowel disease: a prospective 18-month follow-up study. Psychosom Med. 2004;66(1):79-84.

13. Porcelli P, Leoci C, Guerra V, Taylor GJ, Bagby RM. A longitudinal study of alexithymia and psychological distress in inflammatory bowel disease. J Psychosom Res. 1996;41(6):569-573.

14. Persoons $\mathrm{P}$, Vermeire $\mathrm{S}$, Demyttenaere $\mathrm{K}$, et al. The impact of major depressive disorder on the short- and long-term outcome of Crohn's disease treatment with infliximab. Aliment Pharmacol Ther. 2005;22(2):101-110.

15. Mikocka-Walus A, Pittet V, Rossel J-B, et al. Symptoms of depression and anxiety are independently associated with clinical recurrence of inflammatory bowel disease. Clin Gastroenterol Hepatol. 2016;14(6):829-835.

16. North CS, Clouse RE, Spitznagel EL, Alpers DH. The relation of ulcerative colitis to psychiatric factors: a review of findings and methods. Am J Psychiatry. 1990;147(8):974-981.

17. Acosta-Ramirez D, Pagan-Ocasio V, Torres EA, Rodriguez M, Caro O. Profile of the inflammatory bowel disease patient with depressive disorders. PR Heal Sci J. 2001;20(3):215-220.

18. Freitas TH, Andreoulakis E, Alves GS, et al. Associations of sense of coherence with psychological distress and quality of life in inflammatory bowel disease. World J Gastroenterol. 2015;21(21):6713-6727.

19. Goodhand JR, Wahed M, Mawdsley JE, Farmer AD, Aziz Q, Rampton DS. Mood disorders in inflammatory bowel disease: relation to diagnosis, disease activity, perceived stress, and other factors. Inflamm Bowel Dis. 2012;18(12):2301-2309.

20. Nahon S, Lahmek P, Durance C, et al. Risk factors of anxiety and depression in inflammatory bowel disease. Inflamm Bowel Dis. 2012;18(11):2086-2091.

21. Panara AJ, Yarur AJ, Rieders B, et al. The incidence and risk factors for developing depression after being diagnosed with inflammatory bowel disease: a cohort study. Aliment Pharmacol Ther. 2014;39(8):802-810.

22. Howren MB, Lamkin DM, Suls J. Associations of depression with C-reactive protein, IL-1, and IL-6: a meta-analysis. Psychosom Med. 2009;71(2):171-186.

23. Gimeno D, Kivimäki M, Brunner EJ, et al. Associations of C-reactive protein and interleukin- 6 with cognitive symptoms of depression: 12-year follow-up of the Whitehall II study. Psychol Med. 2010;39(3):413-423.

24. Stewart JC, Rand KL, Muldoon MF, Kamarck TW. A prospective evaluation of the directionality of the depression-inflammation relationship. Brain Behav Immun. 2009;23(7):936-944.

25. Reichenberg A, Yirmiya R, Schuld A, et al. Cytokine-associated emotional and cognitive disturbances in humans. Arch Gen Psychiatry. 2001;58(5):445-452.

26. Wright CE, Strike PC, Brydon L, Steptoe A. Acute inflammation and negative mood: mediation by cytokine activation. Brain Behav Immun. 2005;19(4):345-350.

27. Guloksuz S, Wichers M, Kenis G, et al. Depressive symptoms in Crohn's disease: relationship with immune activation and tryptophan availability. PLoS One. 2013;8(3):e60435.

28. Loftus EV, Feagan BG, Colombel J-F, et al. Effects of adalimumab maintenance therapy on health-related quality of life of patients 
with Crohn's disease: patient-reported outcomes of the CHARM trial. Am J Gastroenterol. 2008;103:3132-3141.

29. Minderhoud IM, Samsom M, Oldenburg B. Crohn's disease, fatigue, and infliximab: is there a role for cytokines in the pathogenesis of fatigue? World J Gastroenterol. 2007;13:2089-2093.

30. Bollen J, Trick L, Llewellyn D, et al. The effects of acute inflammation on cognitive functioning and emotional processing in humans: A systematic review of experimental studies. J Psychosom Res. 2017;94:47-55.

31. Clark L, Chamberlain SR, Sahakian BJ. Neurocognitive mechanisms in depression: implications for treatment. Annu Rev Neurosci. 2009;32(1):57-74.

32. Roiser JP, Elliott R, Sahakian BJ. Cognitive mechanisms of treatment in depression. Neuropsychopharmacology. 2012;37(1):117-136.

33. How G. Many subjects does it take to do a regression analysis? Multivariate Behav Res. 1991;26(3):499-510.

34. Kroenke K, Spitzer RL, Williams J. The PHQ-9: Validity of a brief depression severity measure. J Gen Intern Med. 2001;16(9):606-613.

35. Spitzer RL, Kroenke K, Williams J, Löwe B. A brief measure for assessing generalized anxiety disorder: the GAD-7. Arch Intern Med. 2006;166(10):1092-1097.

36. Berkman LF, Blumenthal J, Burg M, et al. Effects of treating depression and low perceived social support on clinical events after myocardial infarction: the Enhancing Recovery in Coronary Heart Disease Patients (ENRICHD) Randomized Trial. JAMA. 2003;289(23):3106-3116.

37. Mitchell PH, Powell L, Blumenthal J, et al. A short social support measure for patients recovering from myocardial infarction: the _ENRICHD_Social Support Inventory. J Cardiopulm Rehabil. 2003;23(6):398-403.

38. Vaglio J, Conard M, Poston WS, et al. Testing the performance of the ENRICHD Social Support Instrument in cardiac patients. Health Qual Life Outcomes. 2004;2(1):24.

39. Brugha T, Bebbington P, Tennant C, Hurry J. The List of Threatening Experiences: a subset of 12 life event categories with considerable long-term contextual threat. Psychol Med. 1985;15(1):189-194.

40. W.H.O. EQ-5D. https://euroqol.org/eq-5d-instruments/eq-5d-5Iabout/. Published 2018.

41. Han S-W, Gregory W, Nylander D, et al. The SIBDQ: further validation in ulcerative colitis patients. Am J Gastroenterol. 2000;95(1):145-151.

42. Irvine EJ, Zhou Q, Thompson AK. The Short Inflammatory Bowel Disease Questionnaire: a quality of life instrument for community physicians managing inflammatory bowel disease. CCRPT Investigators. Canadian Crohn's Relapse Prevention Trial. Am J Gastroenterol. 1996;91(8):1571-1578.
43. Silverberg MS, Satsangi J, Ahmad T, et al. Toward an integrated clinical, molecular and serological classification of inflammatory bowel disease: report of a working party of the 2005 Montreal World Congress of Gastroenterology. Can J Gastroenterol. 2005;19(suppl a): $5 \mathrm{~A}-36 \mathrm{~A}$.

44. 2015 D for C and LG. The English Indices of Deprivation 2015. London.

45. Bland AR, Roiser JP, Mehta MA, et al. EMOTICOM: a neuropsychological test battery to evaluate emotion, motivation, impulsivity, and social cognition. Front Behav Neurosci. 2016;10:25.

46. Baron RM, Kenny DA. The moderator-mediator variable distinction in social psychological research: conceptual, strategic and statistical considerations. J Pers Soc Psychol. 1986;51(6):1173-1182.

47. Preacher KJ, Hayes AF. SPSS and SAS procedures for estimating indirect effects in simple mediation models. Behav Res Methods Instrum Comput. 2004;36(4):717-731.

48. Maxwell S, Cole D. Bias in cross-sectional analyses of longitudinal mediation. Psychol Methods. 2007;12:23-44.

49. Dickens C, Jackson J, Tomenson B, Hay E, Creed F. Association of depression and rheumatoid arthritis. Psychosomatics. 2003;44(3):209-215.

50. Abbott R, Whear R, Nikolaou $\mathrm{V}$, et al. Tumour necrosis factor- $\alpha$ inhibitor therapy in chronic physical illness: A systematic review and meta-analysis of the effect on depression and anxiety. J Psychosom Res. 2015;79(3):175-184.

51. Müller N, Schwarz MJ, Dehning S, et al. The cyclooxygenase-2 inhibitor celecoxib has therapeutic effects in major depression: Results of a double-blind, randomized, placebo controlled, add-on pilot study to reboxetine. Mol Psychiatry. 2006;11(7):680-684.

52. Brown ES. Effects of glucocorticoids on mood, memory, and the hippocampus. Ann N Y Acad Sci. 2009;1179(1):41-55.

53. Agostini A, Filippini N, Cevolani D, et al. Brain functional changes in patients with ulcerative colitis. Inflamm Bowel Dis. 2011;17(8):1769-1777.

How to cite this article: Wilkinson B, Trick L, Knight A, et al. Factors associated with depression in people with inflammatory bowel disease: The relationship between active disease and biases in neurocognitive processing.

Neurogastroenterol Motil. 2019;31:e13647. https://doi. org/10.1111/nmo.13647 